\title{
Healthcare Utilization of Individuals with Opiate Use Disorders: An Analysis of Integrated Medicaid and State Mental Health/Substance Abuse Agency Data
}

\author{
Jeremy Bray, PhD \\ Rita Vandivort, MSW \\ Joan Dilonardo, PhD \\ Laura Dunlap, PhD \\ Don Schroeder, PhD \\ Carol Forhan, MBA \\ Kay Miller, BA
}

\begin{abstract}
Data from the Substance Abuse and Mental Health Services Administration's Integrated Database (IDB) were used to examine the service use patterns of individuals with possible opiate use disorders in Washington State. Results indicate that regardless of Medicaid enrollment status, individuals who received mental health $(M H)$ or substance abuse (SA) services only through state agencies received no inpatient substance abuse service. Furthermore, when compared with individuals who received at least one MH/SA service through Medicaid, those who received services

Address correspondence to Jeremy W. Bray, PhD, Director, Behavioral Health Economics Program, RTI International, 3040 Cornwallis Road, Research Triangle Park, NC 27709, USA. Phone: +1-919-5417003; Fax: +1-919-5416683; E-mail: bray@rti.org.

Rita Vandivort, MSW, Public Health Analyst, Center for Substance Abuse Treatment, Substance Abuse and Mental Health Services Administration, 1 Choke Cherry Road, Rockville, MD 20850, USA. Phone: 1-240-2762974; Fax: +1-2402762960; E-mail: rita.vandivort@samhsa.hhs.gov.

Joan Dilonardo, PhD, Center for Substance Abuse Treatment, Substance Abuse and Mental Health Services Administration, 1 Choke Cherry Road, Rockville, MD 20850, USA. Phone: 1-240-2762974; Fax: +1-240-2762960.

Laura Dunlap, PhD, Research Economist, Behavioral Health Economics Program, RTI International, 3040 Cornwallis Road, Research Triangle Park, NC 27709, USA. Phone: +1-301-8164616; Fax: +1-919-5416683; E-mail: 1jd@rti.org.

Don Schroeder, PhD, Senior Programmer, Thomson/Medstat, 5425 Hollister Avenue, Suite 140, Santa Barbara, CA 93111-2348, USA. Phone: +1-805-6815868; Fax: +1-805-6815888; E-mail: don.schroeder@thomson.com.

Carol Forhan, MBA, Director, Research Data Development, Thomson/Medstat, 5425 Hollister Avenue, Suite 140, Santa Barbara, CA 93111-2348, USA. Phone: +1-805-6815850; Fax: +1-805-6836906; E-mail: carol.forhan@thomson.com.

Kay Miller, BA, Senior Research Manager, Thomson/Medstat, 5425 Hollister Avenue, Suite 140, Santa Barbara, CA 93111-2348, USA. Phone: +1-805-6815855; Fax: +1-805-6815888. E-mail: kay.miller@thomson.com.
\end{abstract}

Journal of Behavioral Health Services \& Research, 2008. (C) 2007 National Council for Community Behavioral Healthcare. 
only through the state agencies were less likely to have received any MH services and were more likely to have received residential SA services. This analysis highlights the importance of using integrated client data in providing a more comprehensive understanding of services to inform policy and raises significant questions about how regulatory requirements affecting different funding mechanisms might drive settings of care in ways not related to the care needed.

\section{Introduction}

The use of, abuse of, and dependence on opiates is a major public health concern. ${ }^{1,2}$ Although only about $0.1 \%$ of the U.S. population reported past-year heroin use in $1998,{ }^{3}$ heroin and other opiate use accounted for about $30 \%$ of total spending for illicit drug use treatment and almost $18 \%$ of spending on drug-related crime. ${ }^{4}$ Psychological and physical health problems were also common among heroin and other opiate users in the mid- to late-1990s. ${ }^{5}$ As recently as 2003 , heroin accounted for $23 \%$ of the mentions of substances used among emergency room patients and $41 \%$ of drug-related deaths recorded by medical examiners or coroners. ${ }^{6}$ The social costs of opiate abuse are not limited to illegal drugs. A recent study suggests that in 2001, the social cost of prescription opioid abuse in the United States was $\$ 8.6$ billion. ${ }^{7}$

Despite the overwhelming societal costs associated with opiate use disorders, few studies have investigated the substance abuse (SA) or mental health $(\mathrm{MH})$ treatment service patterns of individuals suffering from opiate abuse or dependence; rather, most studies focus almost exclusively on those in methadone maintenance treatment. ${ }^{8-10}$ These studies suggest that the co-occurrence of $\mathrm{MH}$ problems and other illicit drug dependence is quite high among individuals with an opiate use disorder. ${ }^{1-13}$ Yet, despite the increased incidence of $\mathrm{MH}$ conditions, individuals with a substance use disorder may not receive adequate levels of $\mathrm{MH}$ care beyond that directly related to their substance use disorder. ${ }^{10,11,14,15}$ Given these findings and the major public health concerns caused by opiate abuse and dependence, the lack of information about the broader $\mathrm{MH} / \mathrm{SA}$ treatment service patterns of individuals with an opiate use disorder is a critical gap in the knowledge base informing policies affecting this population.

This study used data from the Substance Abuse and Mental Health Services Administration's (SAMHSA) Integrated Database (IDB) on individuals in Washington State with an indication of an opiate use disorder to determine what $\mathrm{MH} / \mathrm{SA}$ services they received and through which auspice (i.e., Medicaid or state agency) they received them. Findings from this study offer two contributions to the current literature. First, the IDB presents a unique opportunity to study the behavioral health care utilization of individuals with opiate use disorders because it contains service use data linked at the client level from Washington Medicaid and from the Washington State Department of Social and Health Services' (DSHS) Mental Health Division (MHD) and Division of Alcohol and Substance Abuse (DASA). The combination of Medicaid and DSHS data represented in the IDB provides a more comprehensive picture of service use patterns than might be obtained from studies that focus on only one data source. Second, the late 1990s is often characterized as a time of a heroin epidemic in the United States. ${ }^{16}$ Therefore, examining the $\mathrm{MH} / \mathrm{SA}$ treatment service use patterns of individuals with opiate use disorders during this period can provide an especially relevant baseline for today's policy makers as they attempt to address issues surrounding the emergence of new opiates.

\section{Data and Methods}

This paper uses IDB data on Washington State from three full calendar years (1996-1998). For a more detailed description of the IDB, see Coffey et al., ${ }^{17}$ and for a detailed description of the methods used to link IDB service records across state organizations, see Whalen et al. ${ }^{18}$ 
In addition to service use information, the IDB contains information on patient demographics (e.g., age, gender, race/ethnicity), Medicaid enrollment status, $\mathrm{MH} / \mathrm{SA}$ diagnosis and service codes, and limited provider information.

\section{Study population}

The study population for this analysis consists of individuals who have at least one record in the IDB indicating an opiate use disorder. An opiate use disorder could be indicated by one or more of the following: a diagnosis (either primary or secondary) of an opiate use disorder, a provider type indicative of opiate treatment, a service or procedure code indicative of opiate treatment (including methadone maintenance therapy but excluding methadone used for pain management), or a report of an opioid as the drug of choice. Opiate use disorder diagnoses were defined using the International Classification of Diseases, 9th Revision, Clinical Modification (ICD-9-CM). ICD-9-CM codes of 304.0 or 305.5, including any subclassifications, were used to identify opiate dependence and abuse, respectively. State-specific provider codes were used to identify opiate treatment providers. Both standard [e.g., Healthcare Common Procedure Coding System (HCPCS)] and local procedure codes were used to identify opiate treatment services. Finally, drug-of-choice information was available from self-reports obtained by state agencies during client intake. In some cases, the drug-of-choice information was obtained more than 1 year before or after any SA or MH service provision, but these cases represent less than 3\% of the sample.

Preliminary analyses revealed that very few agency service records include diagnosis codes, but most Medicaid records do. Further investigation revealed that this is not an issue of Medicaid requiring a diagnosis for eligibility, but rather an issue of the data systems themselves. Agency services do not require a diagnosis code for reimbursement, so agency data systems do not track diagnosis. Conversely, Medicaid services often require a diagnosis for reimbursement, so Medicaid data systems track diagnosis. Because the presence or absence of a diagnosis is almost completely confounded by the use or nonuse of Medicaid services, information on service use differences associated with an opiate use disorder diagnosis is not presented.

\section{Client classification}

One of the primary objectives of this study was to examine the extent to which the $\mathrm{MH} / \mathrm{SA}$ service use patterns of individuals with an opiate use disorder varied based on whether their $\mathrm{MH} / \mathrm{SA}$ services were tracked by Medicaid or a state $\mathrm{MH} / \mathrm{SA}$ agency. Importantly, service tracking may, or may not, be associated with full or partial financial coverage. Accordingly, for this study, individuals were classified based on the data source (Medicaid or state $\mathrm{MH} /$ SA agency) from which their IDB $\mathrm{MH} / \mathrm{SA}$ service records were obtained. Based on this information, individuals were classified into three categories:

- Any Medicaid service: individuals who have at least one Medicaid MH/SA service record, regardless of whether they have $\mathrm{MH} / \mathrm{SA}$ state agency service records

- Agency services only with Medicaid enrollment: individuals who have at least one MH/SA state agency service record and were Medicaid-enrolled at some point between their first and last observed $\mathrm{MH} / \mathrm{SA}$ service record but have no record of receiving an $\mathrm{MH} / \mathrm{SA}$ service through Medicaid

- Agency services only without Medicaid enrollment: individuals who have at least one MH/ SA state agency service record and were not Medicaid-enrolled at any time between their first and last observed $\mathrm{MH} / \mathrm{SA}$ service record 
Because the IDB integrates information from state Medicaid and state $\mathrm{MH} / \mathrm{SA}$ agency data sources, overlapping records may occur if Medicaid reimburses a bill, but the state agency provides the service. To avoid overstating utilization rates, only one service date was counted for cases in which the same client, service type (MH or SA), modality/setting of service [i.e., inpatient (IP), residential/long-term care, or outpatient (OP)], and service date were reported on both the Medicaid and state agency databases. Individuals with these types of records were classified as any Medicaid service. Importantly, although individuals in the any Medicaid service group may have received any number, or even the majority, of their services through the state agency, preliminary analyses indicated that individuals who received both Medicaid and agency services were more similar to those who received only Medicaid services than they were to those who received only agency services. For this reason, individuals receiving any Medicaid service were combined into a single category.

In addition to the client categorization described above, the standard IDB client classification was also used. The IDB client classification was used to identify individuals who received services for only $\mathrm{MH}$ conditions ( $\mathrm{MH}$-only), only SA conditions (SA-only), or co-occurring conditions (both $\mathrm{MH}$ and SA) during the study period. The IDB classifies individuals as having co-occurring conditions if they had any of the following within the 3-year study period: (1) both a primary $\mathrm{MH}$ and a SA diagnosis, (2) a primary $\mathrm{MH}$ and a secondary SA diagnosis, or (3) a primary SA and a secondary $\mathrm{MH}$ diagnosis. Individuals classified as having co-occurring conditions did not necessarily have MH and SA conditions concurrently. A client with an $\mathrm{MH}$ record at the beginning of the study period and an SA record at the end of the study period, for example, is classified by the IDB as having co-occurring conditions. In the absence of diagnosis information, $\mathrm{MH}$-only and SA-only classifications were assigned based on the type of service received during the study period (see Coffey et al. ${ }^{17}$ and Bray et al. ${ }^{19}$ for a detailed definition of the primary $\mathrm{MH} / \mathrm{SA}$ diagnosis category).

The IDB client classification does not incorporate information on secondary diagnoses unless a primary $\mathrm{MH} / \mathrm{SA}$ diagnosis is also present, nor does it incorporate drug-of-choice information from state agency intake records. Because both of these pieces of information were used to identify individuals with an opiate use disorder for this study, it is possible for individuals with an opiate use disorder to be classified as $\mathrm{MH}$-only or having received no $\mathrm{MH} / \mathrm{SA}$ service based on the IDB client classification.

\section{Service classification}

Standard IDB service type classifications were used to classify the MH/SA services received by individuals with an opiate use disorder. The IDB $\mathrm{MH} / \mathrm{SA}$ service type classifies $\mathrm{MH} / \mathrm{SA}$ service records as either $\mathrm{MH}$ or SA and within $\mathrm{MH} / \mathrm{SA}$ as IP, residential, or OP. Many inpatient programs are part of psychiatric or general hospitals and generally use a medical model of substance disorders in which intensive medication and counseling are provided over a relatively short period of time. ${ }^{20}$ Residential programs typically are provided in a free-standing, designated residential treatment facility. Residential treatment is usually of longer duration than IP treatment and relies less on medical professionals. Residential treatment provides organized services by designated treatment personnel who provide a planned regimen of care in a $24-\mathrm{h}$ setting and is intended to serve clients who need a safe and stable living environment to develop sufficient recovery skills. ${ }^{21}$ For a complete description of the criteria used to classify services, see Coffey et al. ${ }^{17}$ The vast majority of individuals with a possible opiate use disorder have at least 1 SA service record, but relatively few have MH service records; therefore, the IP, residential, and OP subclassifications were examined for SA service records but not for $\mathrm{MH}$ service records. 


\section{Service encounters}

An individual's total number of service encounter dates was defined as the count of unique dates within the $\mathrm{MH} / \mathrm{SA}$ service window on which the individual had a record with at least one service of a given service category (MH, IP SA, residential SA, or OP SA). Within a single IP or residential stay, each daily service encounter date was counted separately. Using standard IDB definitions for the full SA population, this same information is presented for the broader SA population, excluding individuals with an indication of an opiate use disorder.

\section{Methods}

To characterize the level of contact individuals with an opiate use disorder have with the public treatment system, the analysis examined four key domains: (1) the proportion of clients using services, (2) the median length of the service window (i.e., the length of time between an individual's first and last $\mathrm{MH} / \mathrm{SA}$ service), (3) the number of days of Medicaid enrollment within the service window, and (4) the number of unique $\mathrm{MH} / \mathrm{SA}$ encounter dates within the service window.

Regression analyses were conducted to assess whether the service use patterns of individuals with an opiate use disorder differed significantly across the client classification categories after controlling for differences in the length of the $\mathrm{MH} / \mathrm{SA}$ service window, the length of Medicaid enrollment, and demographics across the client categories. First, logistic regression models of the following form were estimated:

$$
\operatorname{Prob}\left(\operatorname{SERV}_{i}=1\right)=f\left(\beta_{1} \mathrm{DEMOG}_{i}+\beta_{2} \mathrm{GROUP}_{i}+\beta_{3} \mathrm{WINDOW}_{i}+\beta_{4} \mathrm{ENROLL}_{i}\right),
$$

where $\mathrm{SERV}_{i}$ is a series of indicators for receipt of services of a given type. For the regression analyses, any $\mathrm{MH}$ services and $\mathrm{OP}$ and residential SA services were considered. Regression analyses for IP SA services were not conducted because those services were received solely by the any Medicaid service group. DEMOG ${ }_{i}$ is a set of demographic characteristics (i.e., gender, age, and race/ethnicity); GROUP ${ }_{i}$ is a vector of variables representing the data source categories of any Medicaid service (the referent), agency services only with Medicaid enrollment, and agency services only without Medicaid enrollment; WINDOW $_{i}$ is the MH/SA service window length in days; and ENROLL ${ }_{i}$ is the months of Medicaid enrollment (including 0 months). The $\beta$ s are vectors of coefficients to be estimated. Variables reflecting the primary diagnosis categories are not included because much of the information used to classify individuals as $\mathrm{MH}$-only, SA-only, or co-occurring was also used to classify services as $\mathrm{MH}$ or SA.

Next, regressions of the following form were run on days of service, conditional on service use:

$$
\ln \left(\mathrm{DAYS}_{i}\right)=\beta_{1} \mathrm{DEMOG}_{i}+\beta_{2} \mathrm{GROUP}_{i}+\beta_{3} \mathrm{WINDOW}_{i}+\beta_{4} \mathrm{ENROLL}_{i}+\varepsilon_{i},
$$

where DAYS $i$ is a set of variables reflecting the unique days of care for each of the same types of care, and all other terms are as previously defined. Consistent with the recommendations of Manning and Mullahy, ${ }^{22}$ generalized linear model (GLM) estimation with a log link and a gamma distribution on the natural scale was used rather than ordinary least squares (OLS) on the log scale.

\section{Results}

Table 1 presents the demographic characteristics of the individuals identified as having an opiate use disorder in the IDB. For a point of comparison, Table 1 also presents the same information for the general IDB SA population, as defined in Coffey et al., ${ }^{17}$ but excluding 
Table 1

Characteristics of individuals with a possible opiate use disorder compared with the non-opiate

SA population

\begin{tabular}{|c|c|c|}
\hline Population characteristic & Non-opiate SA population & Opiate users \\
\hline All users $(N)$ & 83,793 & 15,652 \\
\hline $\begin{array}{l}\text { Percentage of total } \\
\text { SA population }(\%)\end{array}$ & 84.3 & 15.7 \\
\hline \multicolumn{3}{|l|}{ Gender $(\%)$} \\
\hline Male & 63.2 & 56.8 \\
\hline Female & 36.8 & 43.2 \\
\hline Unknown & 0.0 & 0.0 \\
\hline \multicolumn{3}{|l|}{ Age $(\%)$} \\
\hline Youth $(0-17)$ & 19.9 & 3.1 \\
\hline Adult (18-64) & 78.8 & 96.5 \\
\hline Elderly $(65+)$ & 1.3 & 0.4 \\
\hline Unknown & 0.0 & 0.0 \\
\hline \multicolumn{3}{|l|}{ Race/ethnicity (\%) } \\
\hline White & 70.0 & 77.6 \\
\hline Black & 9.0 & 10.6 \\
\hline Hispanic & 8.5 & 4.3 \\
\hline Native American & 7.8 & 4.5 \\
\hline Other & 3.6 & 2.6 \\
\hline Unknown & 1.0 & 0.3 \\
\hline \multicolumn{3}{|l|}{ IDB client category $(\%)$} \\
\hline SA-only & 68.8 & 59.9 \\
\hline Co-occurring $(\mathrm{MH}+\mathrm{SA})$ & 31.2 & 34.7 \\
\hline MH-only & 0.0 & 1.9 \\
\hline Neither MH nor SA & 0.0 & 3.4 \\
\hline \multicolumn{3}{|l|}{ MH/SA service type (\%) } \\
\hline \multicolumn{3}{|l|}{ SA services $(\%)$} \\
\hline Any setting & 94.9 & 94.6 \\
\hline Inpatient & 3.0 & 6.2 \\
\hline Residential & 31.9 & 57.7 \\
\hline Outpatient & 80.9 & 71.4 \\
\hline No SA services & 5.1 & 5.4 \\
\hline Any MH service & 31.2 & 36.5 \\
\hline \multicolumn{3}{|l|}{$\mathrm{MH} / \mathrm{SA}$ service window length } \\
\hline 25th percentile (days) & 38.0 & 78.0 \\
\hline Median (days) & 168.0 & 348.0 \\
\hline 75th percentile (days) & 446.0 & 770.0 \\
\hline \multicolumn{3}{|l|}{$\mathrm{MH} / \mathrm{SA}$ service data source $(\%)$} \\
\hline Any Medicaid & 46.0 & 51.0 \\
\hline $\begin{array}{l}\text { Agency services only with } \\
\text { Medicaid enrollment }\end{array}$ & 17.3 & 22.7 \\
\hline $\begin{array}{l}\text { Agency services only without } \\
\text { Medicaid enrollment }\end{array}$ & 36.8 & 26.3 \\
\hline
\end{tabular}

SA Substance abuse, $M H$ mental health 
individuals with an opiate use disorder. Individuals with an opiate use disorder accounted for approximately $16 \%$ of the population with any SA disorder. In terms of demographics, when compared with individuals with a non-opiate SA disorder, individuals with an opiate use disorder are less likely to be male and more likely to be adults (as opposed to youth or elderly), but only minor differences in the distribution of clients across racial/ethnic categories are observed. Individuals with an opiate use disorder are less likely to be in the SA-only IDB client classification category. This is largely by construction because as discussed earlier, the current paper uses more inclusive data to identify individuals with a possible opiate use disorder than is used by the IDB client classification. Individuals with an opiate use disorder were more likely to have IP and residential SA services than the general SA population, but less likely to have any OP SA service. Individuals with an opiate use disorder were also more likely to have received any $\mathrm{MH}$ service compared to the general SA population. They also had longer $\mathrm{MH} / \mathrm{SA}$ service windows, indicating longer periods of contact with the public $\mathrm{MH} / \mathrm{SA}$ treatment system, and were more likely to be in the any Medicaid service and the agency services only with Medicaid enrollment groups.

Figure 1 presents the median $\mathrm{MH} / \mathrm{SA}$ service window and length of Medicaid enrollment across individuals with a possible opiate use disorder in each of the data source categories. The any Medicaid service group has the longest median $\mathrm{MH} / \mathrm{SA}$ service window and the most median days of Medicaid enrollment within that window, followed by the agency services

\section{Figure 1}

Median $\mathrm{MH} / \mathrm{SA}$ service window length and days of medicaid enrollment among individuals with a possible opiate use disorder by data source category

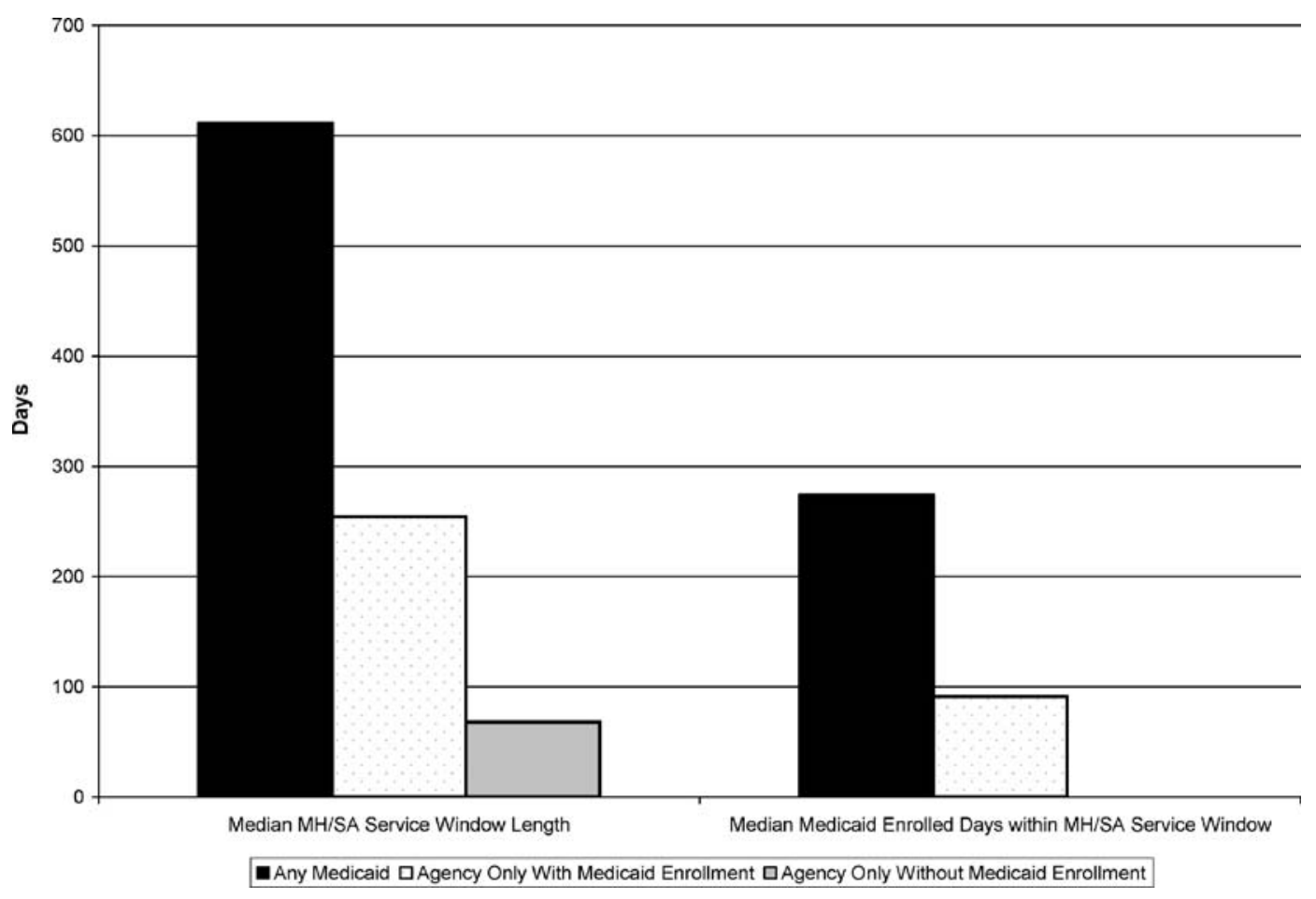


only with Medicaid enrollment group and then the agency services only without Medicaid enrollment group. Because the analysis only examines Medicaid enrollment within the $\mathrm{MH} /$ SA service window, the length of Medicaid enrollment is always less than the total service window length. The total Medicaid enrollment of an individual may be greater than that reported here, but by definition of the service window, no $\mathrm{MH} / \mathrm{SA}$ service use occurred during days of Medicaid enrollment not captured by this measure. Importantly, the any Medicaid service group may have received substantial services from $\mathrm{MH}$ or SA state agencies, because individuals in this group are categorized as any Medicaid service only because they received at least one Medicaid service.

Figures 2 and 3 present the probability of service use and the median days of service conditional on service utilization for each of the data source categories. IP SA care was not included in Figures 2 and 3 because only individuals in the any Medicaid service group had IP SA utilization (the conditional median days of IP SA care for that group were 6). Figure 2 suggests that the two agency services only groups are more likely to receive residential SA services and are less likely to receive OP SA services or MH services. Conditional on receipt of services, Figure 3 suggests that the agency services only with Medicaid enrollment group received the most days of residential SA service, the agency services only without Medicaid enrollment group received the most days of OP SA care, and the any Medicaid services group received the most days of $\mathrm{MH}$ care.

\section{Figure 2}

Percentage of individuals with a possible opiate use disorder using $\mathrm{MH} / \mathrm{SA}$ services by service type and data source category

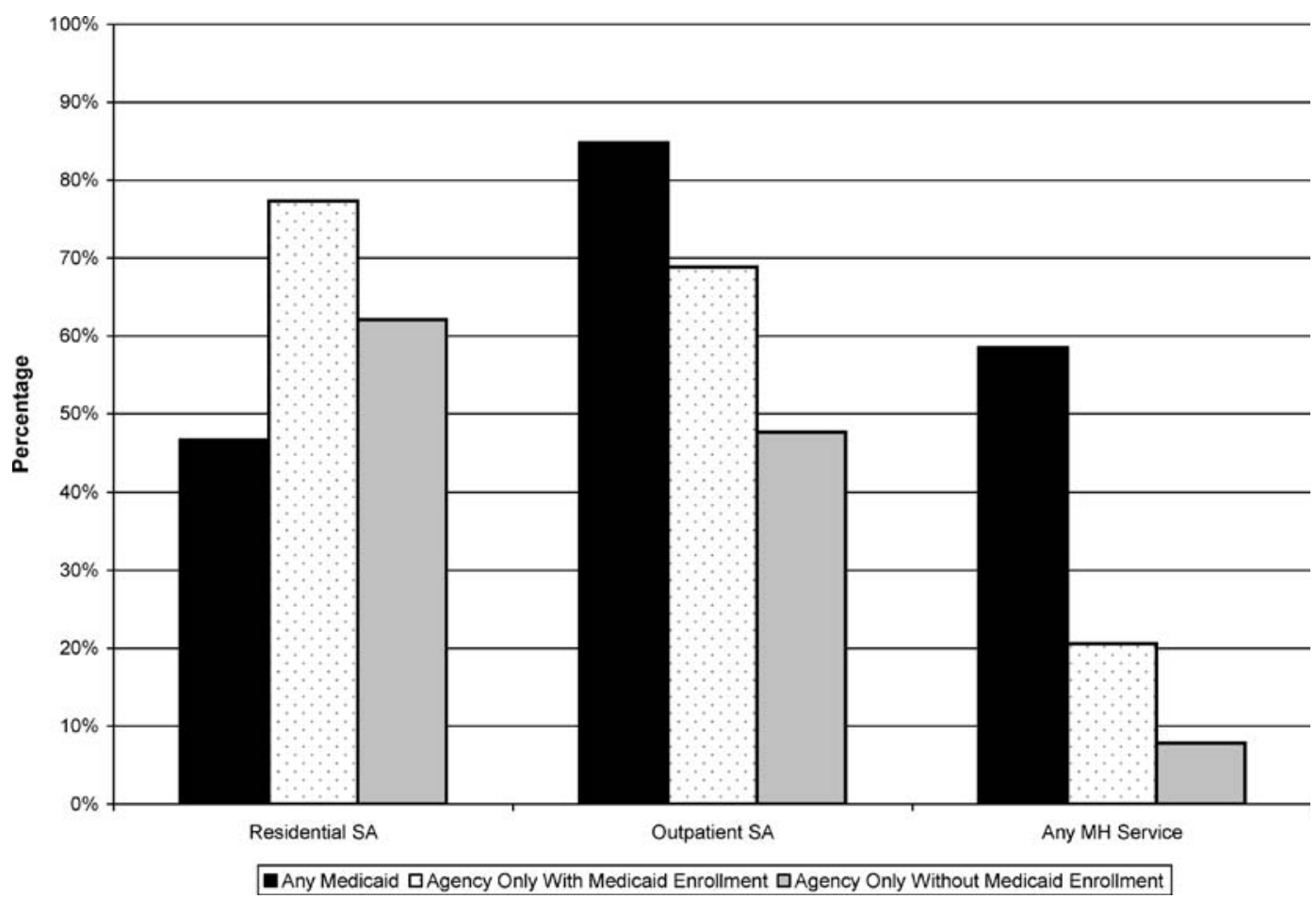




\section{Figure 3}

Median days of care conditional on service use among individuals with a possible opiate use disorder by service type and data source category

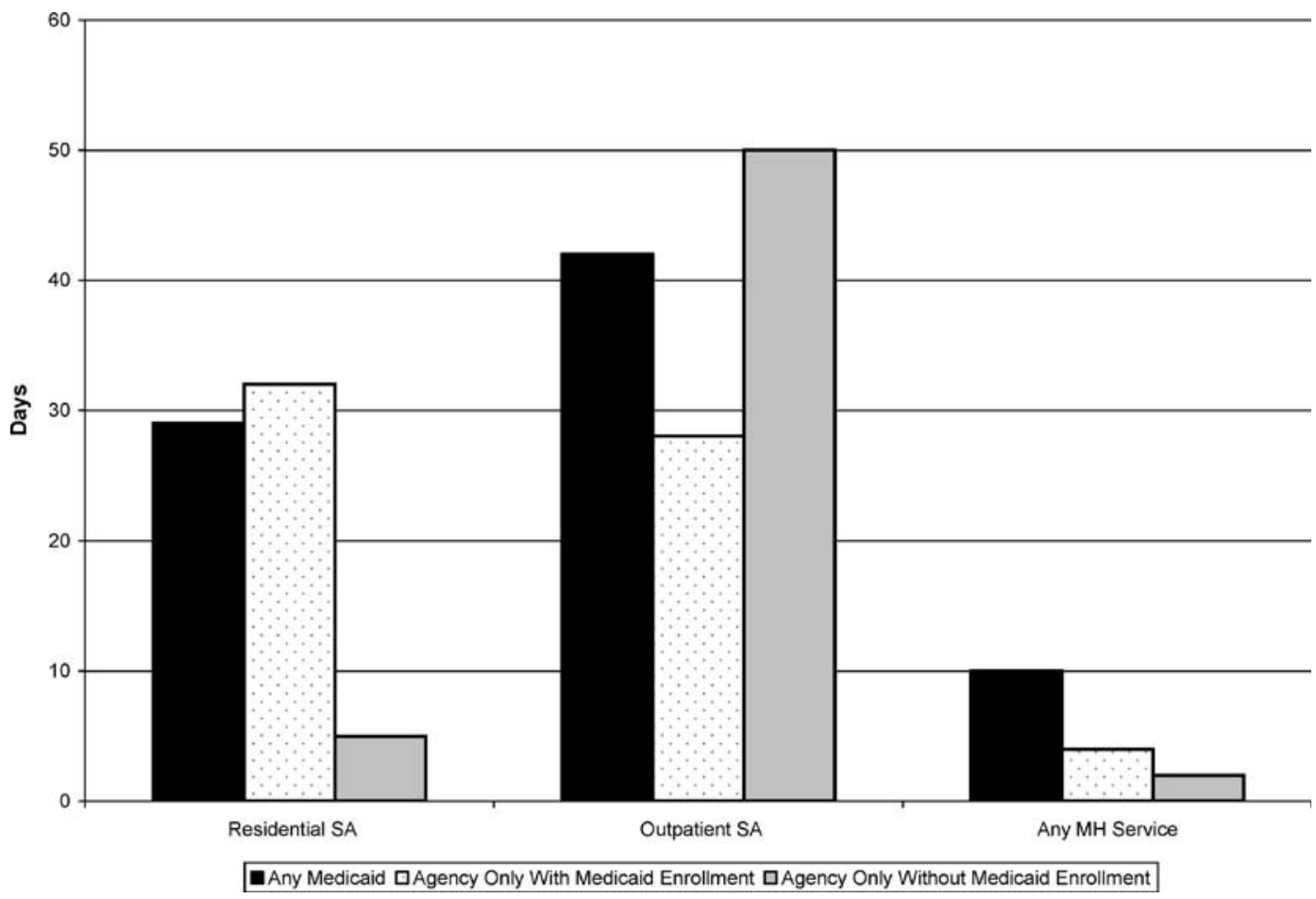

Table 2 presents results from the logistic regressions, including logit coefficients, their standard errors, and associated odds ratios (ORs) for the data source grouping variables. For all other covariates, only the estimated logit coefficients and their standard errors are presented. Table 2 shows that even after controlling for the longer service window and more months of Medicaid enrollment, the pattern observed in Figure 2 still holds. Compared with individuals in the any Medicaid service group, individuals in the agency services only with Medicaid enrollment and the agency services only without Medicaid enrollment groups had significantly higher odds of receiving at least residential SA service $(O R=2.941$ and $\mathrm{OR}=1.376$, respectively) and lower odds of receiving any $\mathrm{OP} S A(\mathrm{OR}=0.311$ and $\mathrm{OR}=0.162$, respectively) or any $\mathrm{MH}$ service $(\mathrm{OR}=0.217$ and $\mathrm{OR}=0.110$, respectively). Briefly examining the results for the control variables in the regression, females are significantly less likely to receive residential SA services. The racial/ethnic categories that are statistically significant suggest that racial/ethnic minorities are less likely than non-Hispanic whites to receive services of any type. Age categories reflecting individuals both younger and older than the referent of 36 to 40 were also, in general, less likely to receive services of any type when the associated coefficients were statistically significant. The exception is that individuals aged 21 to 35 with an opiate use disorder were more likely to receive residential SA services. Results with regard to demographic characteristics, especially race/ethnicity, should not be interpreted as evidence of disparities, however. Rather, they indicate the differential representation of demographic groups across the data and highlight the 


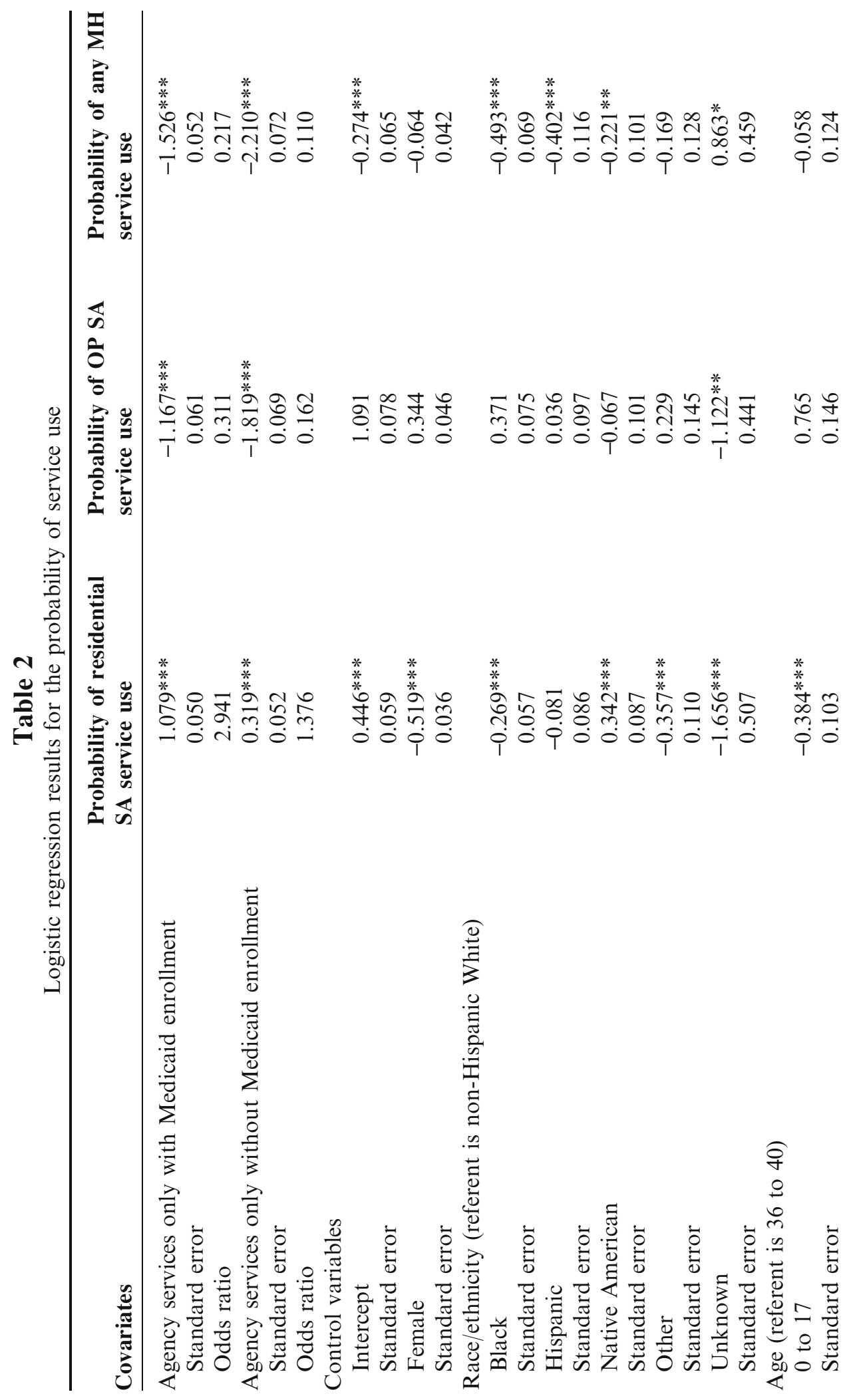




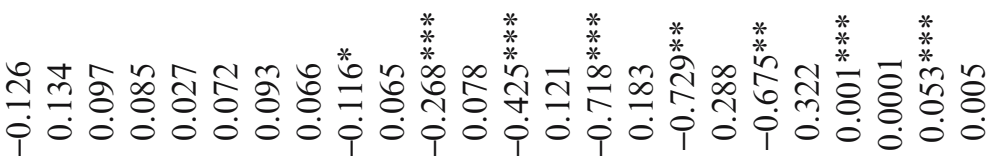

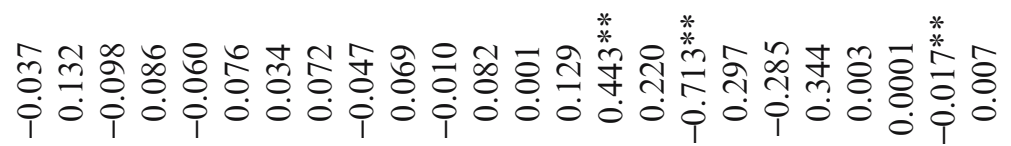

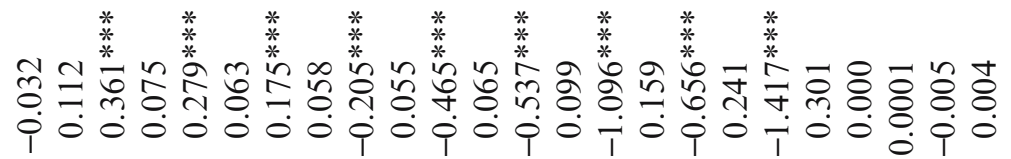

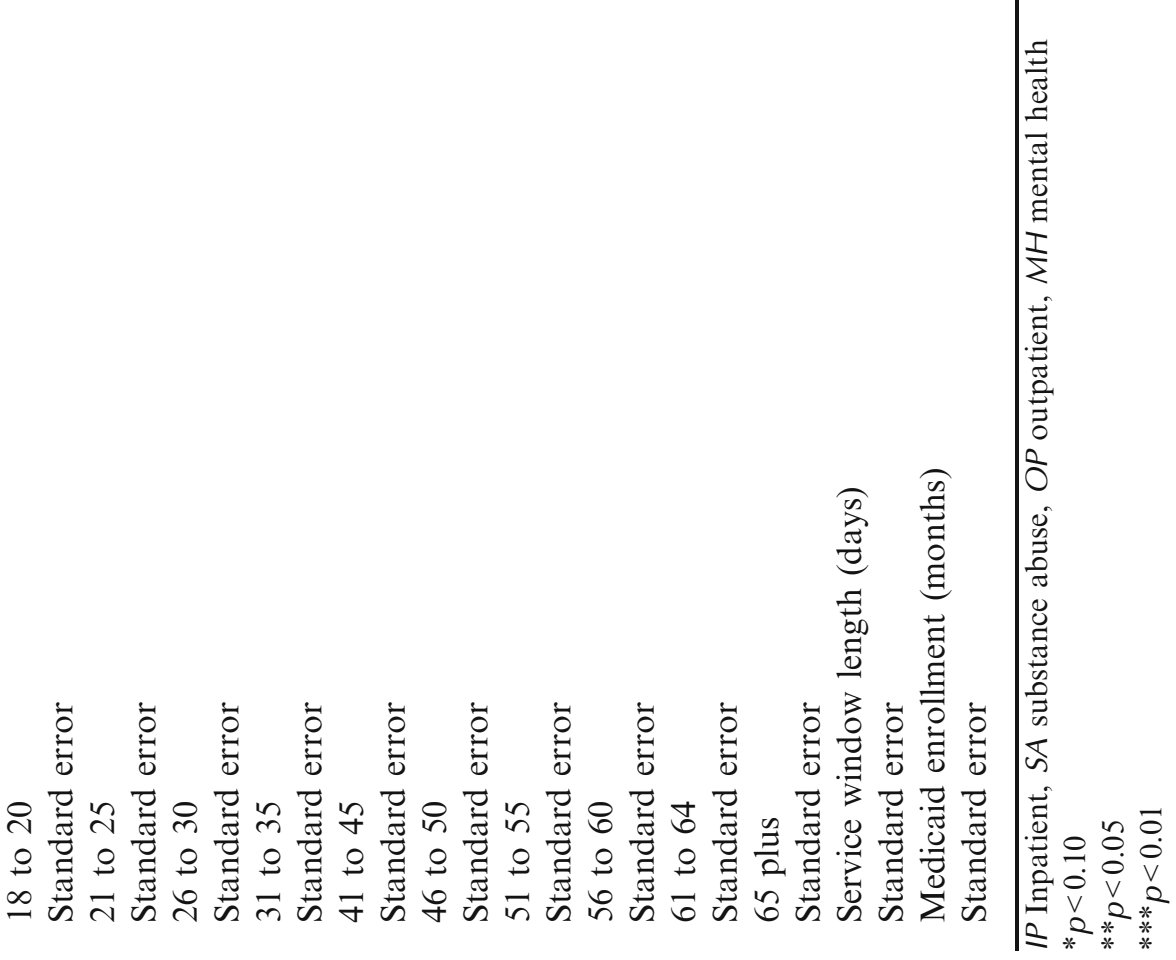


importance of controlling for those factors when assessing statistical significance. Unexpectedly, months of Medicaid enrollment are positively associated with a greater likelihood of service use only for MH services. For both types of SA care examined, longer Medicaid enrollment was negatively associated with the probability of service use, although the relationship was not significant for residential care.

Table 3 presents the regression results for days of care, conditional on having at least 1 day of service of that type of care. Because the natural logarithm of days of care is the dependent variable, the coefficient estimates, standard errors, and associated percentage changes for the data source categories are presented. For all other variables, only the estimated coefficients and their standard errors are presented. For residential SA care, the agency services only with Medicaid enrollment group is significantly associated with more days of residential SA care (28\% increase), while the agency services only without Medicaid enrollment group is significantly associated with fewer days of care (56\% decrease). Both agency services only groups are significantly and positively related to OP SA days of care: an estimated $18 \%$ increase for the agency services only with Medicaid enrollment group and an estimated 60\% increase for the agency services only without Medicaid enrollment group. Finally, the agency services only without Medicaid enrollment group is associated with significantly more days of $\mathrm{MH}$ care than the any Medicaid service group (56\% increase). Unlike in Table 2, no consistent pattern emerges with regard to the demographic control variables included in the linear regression. The length of the $\mathrm{MH} / \mathrm{SA}$ service window is positively and significantly associated with days of care. Months of Medicaid enrollment are negatively and significantly associated with days of residential and OP SA care, but positively and significantly associated with days of $\mathrm{MH}$ care.

\section{Implications for Behavioral Health}

This analysis examined the service use patterns of individuals with an indication of an opiate use disorder using IDB data from Washington for the period 1996 through 1998. Among individuals with opiate use disorders, the receipt of at least one $\mathrm{MH} / \mathrm{SA}$ service through Medicaid appears to be positively associated with IP SA service use in that only individuals who met this condition had any record of an IP SA service. The receipt of at least one $\mathrm{MH} / \mathrm{SA}$ service through Medicaid was also positively associated with OP SA service and with MH service use among individuals with opiate use disorders. The use of only state $\mathrm{MH} / \mathrm{SA}$ agency services, on the other hand, was positively associated with the use of residential SA services.

The findings suggest that the regulatory restrictions faced by state agencies and Medicaid may drive observed patterns of care for individuals with opiate use disorders. Specifically, the finding that agency-only $\mathrm{MH} / \mathrm{SA}$ service use is associated with higher rates of residential service use and with no IP SA service use is likely tied to regulatory differences between Medicaid and the state agencies in Washington. For example, SA block grant funds, which are a key source of funding for both DASA and MHD, could not be used for IP hospital care during the period covered by this analysis. This restriction most likely induced state agencies to route patients for whom outpatient care is insufficient to residential care. Similarly, the Medicaid Institutions of Mental Disorders exclusion prohibits payment for psychiatric services received by adults in residential care facilities with more than 16 beds and so may induce providers to route more severe patients to IP care rather than to residential care. This explanation suggests that using administrative data to track service use patterns may be misleading because the collection of service data is driven by regulatory environments and billing systems that may not capture the actual intensity of care given.

The results are subject to several limitations. First, although the IDB represents one of the most comprehensive cross-system databases used to examine this critical issue to date, it does not 


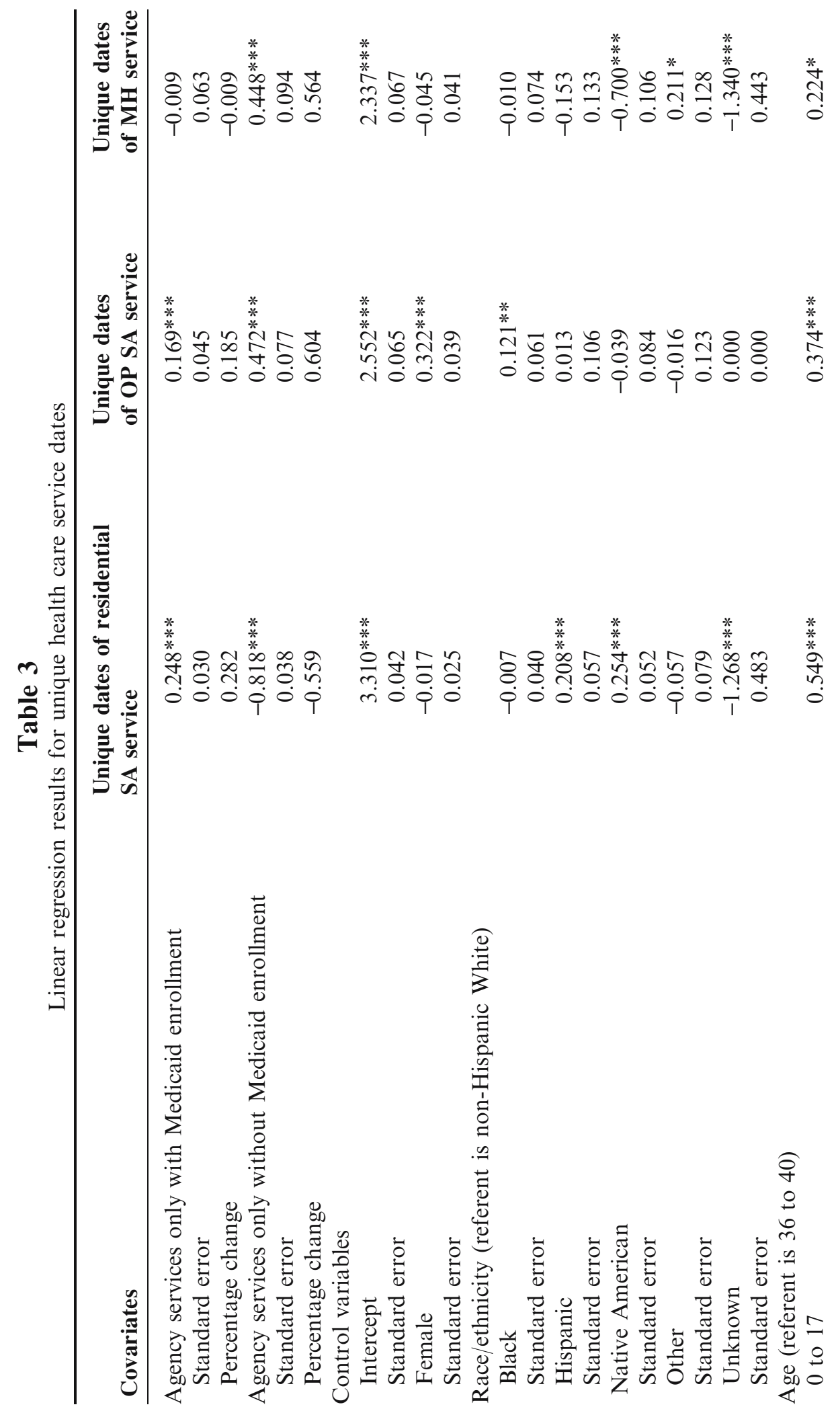




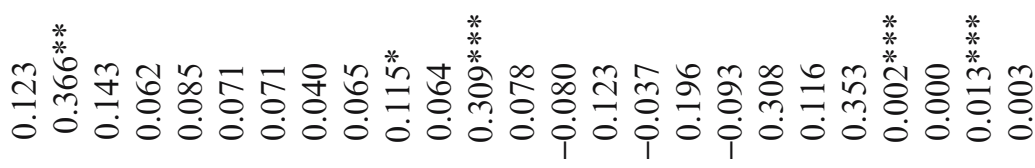

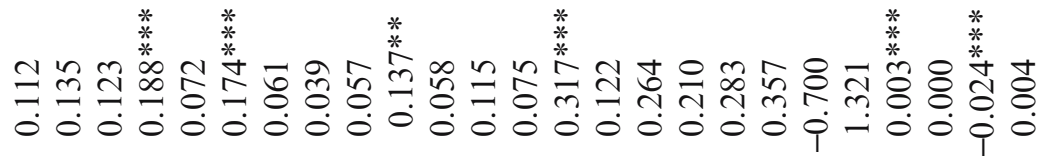

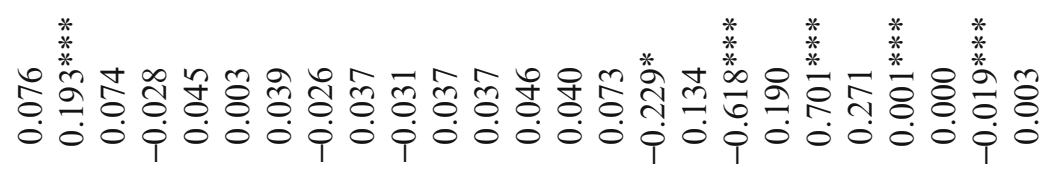

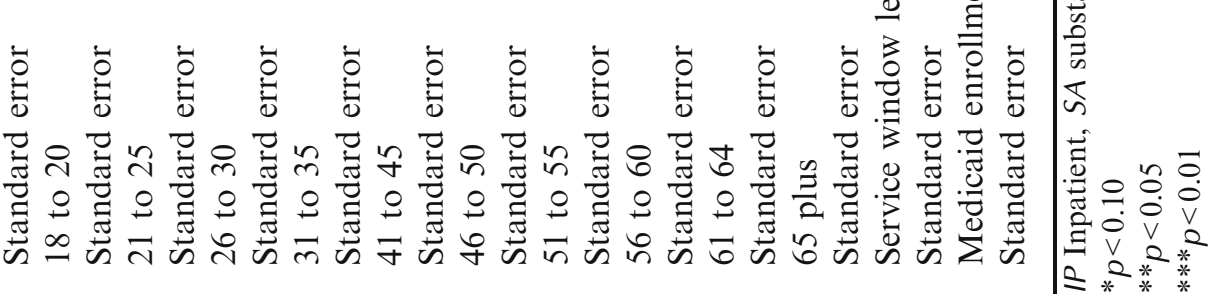


capture all possible services that could be used by individuals with possible opiate use disorders in Washington. Other possible sources include self-pay, private insurance, and the Department of Veterans Affairs (VA) system, among others. Another important limitation common to all administrative databases is that the actual treatment need of the population studied is unknown, so definitive statements cannot be made about the appropriateness of the services received. Furthermore, because of the limited time frame of the IDB, data are unavailable for individuals who used MH/SA services either before 1996 or after 1998. As a result, it is possible that this study has not captured the full service use history of some individuals who appear in the treatment system briefly at the beginning or end of the study period. A final limitation of this study is that information on prescription drug use is not available in the agency service records contained in the IDB and is therefore not considered in this study.

Despite these limitations, the analysis of service use patterns of individuals with possible opiate use disorders during the late 1990s offers key implications for today's policy makers as they attempt to address issues surrounding the emergence of new opiates. First and foremost, if policy makers are trying to track the service use of a small but important segment of the overall SA population, using integrated data is a necessity. When funding for treatment services is cut in an effort to contain costs, it is important to determine if those services have simply been shifted to other state programs, reflecting little net cost savings for states overall. The present study combines state $\mathrm{MH} / \mathrm{SA}$ agency data with state Medicaid data, but including additional data sources (e.g., VA or criminal justice system) would provide an even more complete picture of service use patterns. Second, the utility of administrative data for analyses such as these is often limited by the influence of the regulatory environment, clinical practice patterns, and the institutional history of the data systems used. Given that state and federal policy makers increasingly rely on administrative data to assess the performance of the treatment system, the results clearly highlight the need to better track service provision. SAMHSA's IDB, therefore, represents the vanguard of a new, expansive, cross-agency philosophy regarding administrative data sources and serves as a model for new and more comprehensive data integration efforts.

\section{Acknowledgments}

Funding for this study was provided by the Substance Abuse and Mental Health Services Administration. Valuable comments and suggestions on this research were provided by members of the Integrated Database Technical Expert Panel, which comprises a wide variety of researchers and treatment providers from several state and federal agencies.

\section{References}

1. National Institute on Drug Abuse. Research Report Series. Heroin Abuse and Addiction. NIH Pub. No. 05-4165; May 2005.

2. U.S. Department of Justice. Intelligence Bulletin. OxyContin Diversion, Availability, and Abuse. Product No. 2004-L0424-017; August 2004.

3. Substance Abuse and Mental Health Services Administration, Office of Applied Studies. Results from the 1998 National Household Survey on Drug Abuse. Rockville, MD: Substance Abuse and Mental Health Services Administration, Office of Applied Studies; 2000.

4. Office of National Drug Control Policy (ONDCP). The national drug control strategy 2000 annual report. 2000. Available at: http://www.ncirs.org/ondcppubs/publications/policy/ndcs00/index.html. Accessed August 3, 2005.

5. Ryan CF, White JM. Health status at entry to methadone maintenance treatment using the SF-36 health survey questionnaire. Addiction. 1996;91(1):39-45.

6. Substance Abuse and Mental Health Services Administration, Office of Applied Studies. Drug Abuse Warning Network, 2003: Interim National Estimates of Drug-Related Emergency Department Visits. DAWN Series D-26, DHHS Publication No. (SMA) 043972. Rockville, MD: Substance Abuse and Mental Health Services Administration, Office of Applied Studies; 2004.

7. Birnbaum, HG, White AG, Reynolds JL, et al. Estimated costs of prescription opioid analgesic abuse in the United States in 2001. A societal perspective. Clinical Journal of Pain. 2006;22:667-676. 
8. Deck D, Carlson MJ. Retention in publicly funded methadone maintenance treatment in two western states. Journal of Behavioral Health Services \& Research. 2005;32(1):43-60.

9. Deck D, Carlson MJ. Access to publicly funded methadone maintenance treatment in two western states. Journal of Behavioral Health Services \& Research. 2004;31(2):164-177.

10. Widman M, Platt JJ, Lidz V, et al. Patterns of service use and treatment involvement of methadone maintenance patients. Journal of Substance Abuse Treatment. 1997;14(1):29-35 Jan-Feb.

11. Watkins KE, Burnam A, Kung FY, et al. A national survey of care for persons with co-occurring mental and substance use disorders. Psychiatric Services. 2001;52:1062-1068.

12. Abbott PJ, Weller SB, Walker SR. Psychiatric disorders of opioid addicts entering treatment: preliminary data. Journal of Addictive Diseases. 1994;13(3):1-11.

13. D'Aunno T, Vaughn TE. An organizational analysis of service patterns in outpatient drug abuse treatment units. Journal of Substance Abuse. 1995;7(1):27-42.

14. Stein MD, Friedmann P. Need for medical and psychosocial services among injection drug users: a comparative study of needle exchange and methadone maintenance. The American Journal on Addictions. 2002;11(4):262-270 Fall.

15. Anderson L, Prouty B, Lakin KC. Parallels in size of residential settings and use of Medicaid-financed programs. Mental Retardation. 2000;38(5):468-471 Oct.

16. Kaarlela C. US in midst of heroin epidemic, but many overdose deaths can be prevented. 1999. Available at: http:// www.ucsf.edu/daybreak/1999/04/08_heroin.html.

17. Coffey RM, Graver L, Schroeder D, et al. Mental Health and Substance Abuse Treatment: Results from a Study Integrating Data from State $M H, S A$, and Medicaid Agencies. SAMHSA Publication No. SMA-01-3528. Rockville, MD: Center for Substance Abuse Treatment and Center for Mental Health Services, Substance Abuse and Mental Health Services Administration; 2001.

18. Whalen D, Pepitone A, Graver L, et al. Linking Client Records from Substance Abuse, Mental Health and Medicaid State Agencies. SAMHSA Publication No. SMA-01-3500. Rockville, MD: Center for Substance Abuse Treatment and Center for Mental Health Services, Substance Abuse and Mental Health Services Administration; July 2001.

19. Bray JW, Davis KL, Graver L, et al. Mental health and substance abuse treatment utilization among individuals served by multiple public agencies in three states. Journal of Behavioral Health Services and Research. 2005;32(3):282-293.

20. Mojtabai R, Zivin JG. Effectiveness and cost-effectiveness of four treatment modalities for substance disorders: a propensity score analysis. Health Services Research. 2003;38(1p1):233-259.

21. American Society of Addiction Medicine. Patient Placement Criteria for the Treatment of Substance-Related Disorders, 2 nd Edition (ASAM PPC-2). Chevy Chase, MD: American Society of Addiction Medicine; 1996.

22. Manning WG, Mullahy J. Estimating log models: to transform or not to transform? Journal of Health Economics 2001;20:461-494. 